\title{
Gerenciamento do tempo no processo de trabalho dos enfermeiros em Unidade de Terapia Intensiva
}

Time management in the work process of nurses in the intensive care unit

Gestión del tiempo en el proceso de trabajo de enfermeros de la unidad de cuidados intensivos

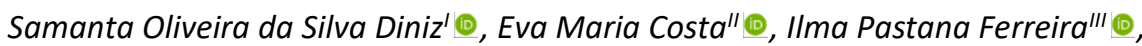 \\ Paulo Sérgio da Silva ${ }^{\prime V}{ }^{\circledR}$, Teresa Tonini" ${ }^{\circledR}$
}

'Universidade Unigranrio, Duque de Caxias, RJ, Brasil; "Universidade Federal do Estado do Rio de Janeiro, Rio de Janeiro, RJ, Brasil; "I'Universidade do Estado do Pará, Belém, PA, Brasil; "Universidade Federal de Roraima, Boa Vista, RR, Brasil

\begin{abstract}
RESUMO
Objetivo: analisar o gerenciamento do tempo dispensado por enfermeiros em intervenções de cuidados diretos e indiretos, em atividades associadas ao trabalho e atividades pessoais. Método: estudo quantitativo, transversal, realizado em Unidade de Terapia Intensiva de um hospital universitário no Rio de Janeiro em janeiro de 2017. Foram realizadas observações diretas das atividades realizadas pelos enfermeiros em 18 plantões, totalizando 216 horas. $O$ "Instrumento para mensuração da carga de trabalho" foi utilizado para estruturar a observação. Resultados: os resultados mostraram que $21,5 \%$ do tempo dos enfermeiros foram dedicados às intervenções de cuidados diretos $44,7 \%$ aos cuidados indiretos, $6,1 \%$ às atividades associadas e $27,7 \%$ às atividades pessoais. Conclusão: os enfermeiros utilizam maior parte do tempo em atividades não relacionadas ao cuidado direto ao paciente. Os achados podem ser utilizados pelos gestores para revisão e adequação do dimensionamento de profissionais na assistência direta e indireta e do processo de trabalho na Unidade.

Descritores: Unidades de Terapia Intensiva; Recursos Humanos de Enfermagem; Cuidados de Enfermagem; Gerenciamento do Tempo.
\end{abstract}

\section{ABSTRACT}

Objective: to analyze time management by nurses in direct and indirect care interventions, in work-related and personal activities. Method: this quantitative, cross-sectional study was conducted in the Intensive Care Unit of a university hospital in Rio de Janeiro in January 2017. Direct observations were made of nurses' activities in 18 shifts, totaling 216 hours. Mello's "Workload Measuring Tool" was used to structure the observations. Results: $21.5 \%$ of nurses' time was devoted to direct care, $44.7 \%$ to indirect care, $6.1 \%$ to work-related activities and $27.7 \%$ to personal activities. Conclusion: nurses spend most of their time on activities unrelated to direct patient care. These findings can be useful to managers in reviewing and adjusting both staffing in direct and indirect care and the unit's work process.

Descriptors: Intensive Care Units; Nursing Staff; Nursing Care; Time Management.

\section{RESUMEN}

Objetivo: analizar la gestión del tiempo que brindan los enfermeros en las intervenciones asistenciales directas e indirectas, en actividades asociadas al trabajo y actividades personales. Método: estudio cuantitativo, transversal, realizado en la Unidad de Cuidados Intensivos de un hospital universitario de Rio de Janeiro en enero de 2017. Se realizaron observaciones directas de las actividades realizadas por enfermeras en 18 turnos, totalizando 216 horas. Se utilizó la "Herramienta de medición de la carga de trabajo" para estructurar la observación. Resultados: los resultados mostraron que el $21,5 \%$ del tiempo de los enfermeros se dedicó a intervenciones de cuidados directos, el $44,7 \%$ a cuidados indirectos, el $6,1 \%$ a actividades asociadas y el $27,7 \%$ a actividades personales. Conclusión: los enfermeros dedican la mayor parte de su tiempo a actividades no relacionadas con la atención directa al paciente. Los hallazgos pueden ser utilizados por los gestores para revisar y adecuar el dimensionamiento de profesionales en la atención directa e indirecta y el proceso de trabajo en la Unidad.

Descriptores: Unidades de Cuidados Intensivos; Personal de Enfermería; Atención de Enfermería; Administración del Tiempo.

\section{INTRODUÇÃO}

O conceito de tempo assume perspectivas distintas, porque os seres humanos são dotados de variadas experiências e objetivos. Com a evolução da sociedade, a concepção do tempo percorreu múltiplos estágios passando desde a condição cíclica, descontínua e qualitativa, quando o homem utilizava os movimentos do sol, da lua e das estrelas como instrumentos de medição de tempo, até assumir o sentido linear, progressivo e quantitativo com a sincronização através de relógio. Entretanto, somente com a revolução industrial que se estabeleceram, de fato, novas relações entre o tempo e o trabalho ${ }^{1,2}$.

Artigo extraído da tese de doutorado "Gerenciamento do tempo e carga de trabalho em Unidade de Terapia Intensiva: implicações nos registros dos enfermeiros" (Programa de Pós-Graduação em Enfermagem e Biociências, da Universidade Federal do Estado do Rio de Janeiro).

Autora correspondente: Samanta Oliveira da Silva Diniz. E-mail: rmos_sam@yahoo.com.br

Editora Científica: Cristiane Helena Gallasch 
O estudo do tempo no processo de trabalho das Organizações foi fundamental para o seu crescimento e para o controle de custos. As instituições de saúde, como qualquer outra organização, carecem de estratégias que tenham a capacidade de subsidiar as tomadas de decisão na busca pelo aumento da qualidade, a melhoria da produtividade e a otimização de recursos. Contudo, no contexto do trabalho em saúde, existem elementos consideráveis que dificultam a determinação do tempo efetivo de trabalho².

Estudos que objetivem investigar e mensurar o tempo efetivo de trabalho dos profissionais de saúde são de extrema relevância. As instituições de saúde passaram por processo intenso de desenvolvimento, em que necessárias mudanças ocorreram na estrutura, como o uso de aparatos altamente complexos, e nos processos de trabalho assistenciais, gerenciais e organizacionais relacionados à capacitação de recursos humanos e à avaliação da qualidade dos cuidados prestados.

A velocidade das mudanças, que marcam o contexto atual do processo de trabalho em saúde, exige respostas diferenciadas dos indivíduos e das instituições, no que diz respeito à efetividade dos serviços prestados. No âmbito da enfermagem, o alcance dos resultados está associado às questões do microespaço pertinente ao cotidiano da prática de cuidar e gerenciar ${ }^{3}$.

Processo de trabalho é a transformação, intencional e consciente, do objeto em produto, por meio da intervenção do ser humano que, para fazê-lo, emprega instrumentos ${ }^{4-6}$. O processo de trabalho do enfermeiro, desde a profissionalização da enfermagem, envolve o cuidar - ações de cuidado de forma direta - e o administrar - ações de cuidado de forma indireta ${ }^{7}$.

As intervenções de cuidado de forma direta são aquelas realizadas por meio da interação com o cliente e ações práticas, incluindo cuidados de enfermagem de caráter fisiológico, psicossocial, apoio e aconselhamento. As intervenções de cuidados de forma indireta são aquelas ações realizadas à distância, mas em benefício do cliente e abrangem práticas direcionadas ao gerenciamento do processo de trabalho e interação multiprofissional ${ }^{8}$.

O enfermeiro, no espaço da Unidade de Terapia Intensiva (UTI), possui múltiplas atribuições que envolvem o cuidado de forma direta e indireta, atividades burocráticas e gerenciais. Inúmeros fatores podem influenciar na forma como o enfermeiro gerencia o seu tempo, tais como número reduzido de profissionais para atender as demandas, sucateamento das Instituições públicas de saúde e precariedade dos recursos materiais destinados à assistência.

O cuidado de enfermagem em UTI exige do enfermeiro o enfrentamento de inúmeros desafios estruturais e processuais, entre eles: o crescente grau de exigência técnico-científica devido ao aumento da complexidade das afecções orgânicas dos clientes, dos aparatos tecnológicos utilizados para o tratamento e das múltiplas possibilidades de intervenção; a necessidade de controle de qualidade da assistência prestada, mesmo diante de dificuldades enfrentadas por falta de materiais/insumos para o tratamento/cuidado ao cliente; a falta de valorização do profissional por parte das instituições e o número reduzido de profissionais de enfermagem frente à complexidade de cuidados exigidos pelos clientes em UTI que gera sobrecarga de trabalho da equipe ${ }^{9}$.

Embora existam estudos com análises da distribuição do tempo de trabalho dos enfermeiros em diversos ambientes, ${ }^{10-14}$ torna-se premente o aprofundamento de pesquisas em UTIs a fim de se mapear o processo de trabalho dos enfermeiros neste espaço de cuidar.

O estudo se justifica pela necessidade em avaliar a distribuição do tempo e o movimento do corpo dos enfermeiros no ambiente de alta complexidade, pois são elementos fundamentais para se compreender as dificuldades enfrentadas e estratégias adotadas por eles no cotidiano da prática de cuidar e gerenciar.

A relevância do estudo se concentra na possibilidade de elucidação sobre a forma como o enfermeiro planeja, organiza, administra, monitora e avalia as atividades no seu processo de trabalho. Ademais, localiza intervenções de enfermagem, o tempo dispensado em cada intervenção, a distribuição do tempo pelo enfermeiro entre as ações de cuidado direto e indireto, contribuindo para a construção do conhecimento na área da gestão em saúde, organização dos serviços de enfermagem e planejamento das dinâmicas assistenciais, sobretudo em ambientes altamente tecnificados.

Nesta perspectiva, teve-se como objetivo analisar o gerenciamento do tempo dispensado por enfermeiros em intervenções de cuidados diretos e indiretos, em atividades associadas ao trabalho e atividades pessoais.

\section{MÉTOdo}

Trata-se de estudo exploratório transversal, desenvolvido na UTI de um hospital universitário (HU) localizado no estado do Rio de Janeiro. A unidade possui 10 leitos ativos que atendem clientes clínicos e cirúrgicos. 0 quadro de enfermeiros é constituído por um coordenador e 12 enfermeiros assistenciais. 
Foram incluídos no estudo os 12 enfermeiros assistenciais, dispensando desta forma processos de amostragem. Os critérios de exclusão foram enfermeiros remanejados de outros setores da instituição que estivessem atuando na UTI no dia da coleta de dados. Os participantes foram caracterizados quanto ao sexo, idade, tempo de trabalho na instituição, tempo de formação profissional e maior qualificação profissional.

Após assinatura do Termo de Consentimento Livre e Esclarecido (TCLE), os dados foram coletados por meio de observações diretas das atividades realizadas pelos enfermeiros em 18 plantões, sendo nove plantões diurnos e nove plantões noturnos, totalizando 216 horas de observação.

Para o levantamento do tempo dispensado pelos enfermeiros nas atividades, utilizou-se a observação sistemática, direta e não participante. O "Instrumento para mensuração da carga de trabalho" 15 é composto por sistema categorial e de checagem e foi adotado para estruturar a observação. O Sistema categorial de Mello (2011) é dividido em três categorias, a saber: Categoria I - Cuidados de enfermagem: são contemplados 7 domínios, 24 classes e 126 intervenções de enfermagem da Classificação das Intervenções de Enfermagem (NIC); categoria II - Atividades associadas: são contempladas 14 atividades assumidas pela equipe de enfermagem, mas que poderiam ser realizadas por outras categorias profissionais e categoria III - Atividades pessoais: são atividades relacionadas às necessidades pessoais do profissional.

O sistema de checagem contém 36 espaços para o registro do observador, com intervalos de 10 minutos, isto é, no turno da manhã (7h-13h), os espaços foram identificados como 7h, 7h10, 7h20, 7h30 e, assim, sucessivamente até 12h50. Da mesma forma, com o turno da tarde (13h-18h50). À noite, o período é dividido em noturno 1 (19h-24h50) e noturno 2 (1h-6h50).

Nos nove plantões diurnos, a coleta de dados ocorreu das $7 \mathrm{~h}$ às $19 \mathrm{~h}$ e nos nove plantões noturnos a coleta foi das $19 \mathrm{~h}$ às $7 \mathrm{~h}$. Como a coleta se deu de forma ininterrupta em plantões de $12 \mathrm{~h}$, foram recrutados observadores. Por acreditar que a coleta de dados seria mais confiável se fosse realizada por pessoas com formação na área de enfermagem, buscou-se estudantes do curso de graduação em enfermagem.

O grupo de observadores foi constituído por oito estudantes do décimo período de enfermagem de uma universidade privada do Rio de Janeiro, que receberam dez horas de treinamentos teórico e prático para garantir fidedignidade na aplicação do instrumento de coleta. Ademais, a pesquisadora foi uma das observadoras e esteve presente em todo o período da coleta de dados, elucidando possíveis dúvidas.

A escala de observadores foi de dois por turno para não interferir na dinâmica do trabalho da equipe. No início de cada turno, os observadores recebiam os instrumentos impressos a serem utilizados naquele turno e ajustavam seus relógios para que as medidas ocorressem no mesmo momento.

Para cada enfermeiro observado, utilizou-se um impresso diferente. Ressalta-se a necessidade, no primeiro momento, da identificação do instrumento com o nome do enfermeiro, pois foi utilizado o mesmo impresso durante todo o turno de observação. A cada 10 minutos, o observador assinalava a intervenção/atividade que estava sendo realizada pelo enfermeiro. Posteriormente, para a análise dos dados, o nome do profissional foi substituído por um número, a fim de preservar o anonimato dos participantes. Cada observador acompanhou apenas um enfermeiro por turno, para garantir que nenhuma intervenção/atividade fosse perdida.

Os dados foram digitados, armazenados e processados utilizando o programa Microsoft Excel ${ }^{\circledR}$. A análise possibilitou levantar a distribuição percentual do tempo despendido pelos enfermeiros nas Intervenções de NIC e a distribuição percentual de tempo destinado às atividades de cuidado de forma direta e indireta, atividades associadas e atividades pessoais.

O projeto de pesquisa foi submetido e aprovado pelo Comitê de Ética em Pesquisa da Universidade correspondente respeitando a normatização da Resolução № 466/2012.

\section{RESULTADOS}

A Tabela 1 mostra a distribuição proporcional da quantificação do tempo dos enfermeiros utilizado nas intervenções de NIC, atividades associadas e atividades pessoais.

Os resultados mostram que maior número de intervenções de NIC correspondeu ao domínio 6 referentes às intervenções que oferecem suporte ao uso do sistema de saúde (38\%), seguidas das intervenções do domínio fisiológico complexo (16,7), fisiológico básico (8\%), segurança $(2,4 \%)$ e comportamental (1\%). As atividades de NIC dos domínios 5 (Família) e 7 (Comunidade) não foram realizadas pelos enfermeiros no período de coleta de dados.

As atividades associadas representaram 6,2\% do tempo dos enfermeiros e corresponderam àquelas assumidas pela equipe de enfermagem e que poderiam ser realizadas por outras categorias profissionais. As atividades pessoais ocuparam $27,7 \%$ do tempo. 
TABELA 1: Distribuição proporcional da quantificação do tempo dos enfermeiros utilizado nas intervenções de NIC, atividades associadas e atividades pessoais na UTI. Rio de Janeiro, RJ, Brasil, 2017

\begin{tabular}{ll}
\hline Intervenções de NIC, atividades associadas e atividades pessoais & Tempo (\%) \\
\hline Domínio 1- fisiológico básico & 8 \\
Domínio 2- fisiológico complexo & 16,7 \\
Domínio 3- comportamental & 1 \\
Domínio 4- segurança & 2,4 \\
Domínio 5- família & 0 \\
Domínio 6- sistema de saúde & 38 \\
Domínio 7- comunidade & 0 \\
Atividades associadas & 6,2 \\
Atividades pessoais & 27,7 \\
\hline
\end{tabular}

A Tabela 2 apresenta a distribuição do tempo dos enfermeiros, nas atividades de cuidados de forma direta, indireta, atividades associadas e pessoais.

\begin{tabular}{|c|c|}
\hline $\begin{array}{l}\text { Cuidados de forma direta e indireta, atividades } \\
\text { associadas e atividades pessoais }\end{array}$ & Tempo (\%) \\
\hline Cuidados de forma direta & 21,5 \\
\hline Cuidados de forma indireta & 44,7 \\
\hline Atividades associadas & 6,1 \\
\hline Atividades pessoais & 27,7 \\
\hline
\end{tabular}

Os resultados mostram que $44,7 \%$ do tempo dos enfermeiros são dedicados aos cuidados indiretos, 21,5 aos cuidados diretos, 6,1 as atividades associadas e 27,7 as atividades pessoais.

\section{DISCUSSÃO}

Os resultados evidenciados mostram semelhanças com outros estudos desenvolvidos sobre a mesma temática. Quanto à distribuição do tempo pelos enfermeiros, o domínio 1, fisiológico básico, requereu $8 \%$ do tempo dos enfermeiros, resultado diferente de outros estudos em que os percentuais foram de $20 \%{ }^{15}$ e $36 \%{ }^{16}$.

A intervenção com maior representatividade, neste domínio, foi o banho (3,2\%). Resultados semelhantes foram encontrados em outros estudos em que o banho correspondeu a $1,4 \%^{16}$ e $5,4 \%{ }^{17}$, do tempo da equipe de enfermagem.

Mesmo sendo a atividade predominante no domínio 1, os estudos mostraram que a participação do enfermeiro no banho no leito ainda é insignificante, quando se trata do cuidado ao cliente crítico. A participação no banho oportuniza os enfermeiros a estabelecer maior interação e comunicação e possibilita avaliação mais minuciosa do cliente.

O domínio 2, fisiológico complexo, correspondeu a 16,7\% do tempo dos enfermeiros. Outros estudos encontraram valores mais expressivos com percentuais de $19,4 \%{ }^{15}$ e $15 \%{ }^{16}$. A maior parte do tempo despendido pelos enfermeiros, nas intervenções do domínio 2 de NIC, corresponde a controle hidroeletrolítico (8,3\%) e administração de medicamentos $(6,9 \%)$.

No que se refere ao controle hidroeletrolítico, quase todas as ações se relacionavam com o registro da ingestão/administração e eliminação dos clientes no balanço hídrico. Na unidade, os enfermeiros são responsáveis por esse controle, seja no preenchimento dos dados seja na conferência/somatório do balanço no final do plantão.

Quanto à administração de medicamentos, verificou-se que, em diversos plantões, o enfermeiro assumia a responsabilidade pelo preparo e administração das medicações de todos os clientes, enquanto a equipe técnica realizava a higiene no leito e os curativos. Com isso, o enfermeiro além de não participar do banho, não conseguia avaliar as lesões e os locais de incisão.

O número reduzido de profissionais levou os enfermeiros a delegarem à equipe técnica, atribuições que deveriam ser realizadas por ele, considerando o fato de que o cliente se encontra em estado crítico. A comparação com outros estudos sobre a temática mostra que a administração de medicamentos correspondeu a $13,3 \%{ }^{16}$ e $5,9 \%{ }^{17}$ do tempo da equipe de enfermagem, respectivamente. 
O percentual de tempo dedicado às intervenções do domínio 3 foi de apenas 1\%. Em estudo similar ${ }^{15}$, o domínio 3 foi encontrado em apenas 0,2\%, fortalecendo o achado do estudo atual. Das atividades, a mais frequente foi escutar ativamente. Pode-se inferir que a razão para o enfermeiro não dedicar mais tempo às intervenções desse domínio na UTI seja pelo fato de quase todos os clientes apresentarem alteração de nível de consciência, seja pelo coma ou pelo uso de sedativos.

O domínio 4, segurança, representou 2,4\% de todas as atividades. Estudos com a mesma temática encontraram percentuais superiores $10,7 \%{ }^{15}$ e $9 \%{ }^{16}$. A intervenção mais frequente foi monitorização dos sinais vitais $(1,4 \%)$. 0 achado é inquietante, pois as intervenções deste domínio se relacionam aos cuidados que dão suporte à proteção contra danos. Por ser clientes que exigem cuidados intensivos, se esperava maior tempo de dedicação dos enfermeiros a essas atividades.

As atividades correspondentes ao domínio 5, família, não foram realizadas pelos enfermeiros no período de coleta de dados. Outros estudos sobre o mesmo fenômeno apresentaram baixo percentual de atividades relacionadas ao domínio "família", 0,5\% ${ }^{15}, 4 \%{ }^{16}$ e 1,13\% ${ }^{17}$, contudo não apresentaram ausência de intervenções como no estudo atual. Ainda que a presença da família na UTI seja restrita ao horário de visita, a ausência de ações neste domínio aponta para a falta de interação/comunicação entre o enfermeiro e os familiares, resultado também encontrado em outros estudos $^{18}$. A comunicação é o caminho mais seguro para se estabelecer a relação de ajuda e se criar vínculo e acolhimento da enfermagem com cliente-família ${ }^{19}$

O domínio 6, Sistema de saúde, que abarca as intervenções que subsidiam o uso do sistema de atendimento em saúde, foi o de maior representatividade nas UTIs (38\%). Estudos realizados no centro cirúrgico, pediatria, unidade básica de saúde e UTI, ou seja, em cenários diversificados, o domínio 6 também apresentou importante representatividade com 22,12\%, 30\%, 54\% e 24,1\%, respectivamente ${ }^{15-16,20}$. Esse dado permite afirmar que, independente do cenário, as intervenções relacionadas a este domínio despendem mais de $20 \%$ do tempo dos profissionais de enfermagem, especialmente, dos enfermeiros. Em alguns estudos, tanto quanto neste, as atividades desse domínio são as mais representativas ${ }^{15,20}$.

As atividades, do domínio 6, mais frequentemente encontradas foram controle de suprimentos (11,1\%) e documentação $(36,7 \%)$

No que se refere ao controle de suprimentos, as atividades mais comuns foram: solicitação de materiais e medicamentos, recebimento de material e medicamento recebido e conferência de materiais da unidade. Os enfermeiros passam grande parte do tempo, conferindo materiais do setor com o uso de impressos e checklists padronizados pela unidade.

Do tempo dedicado à documentação, $52 \%$ foram destinados aos registros no prontuário do cliente, os outros $48 \%$ do tempo foram reservados às anotações secundárias em livros de ordens e ocorrências, livros de controle de entrada e saída de pacientes e livros de controle de exames. Outros estudos mostram que o enfermeiro dedica 22,93\%, 20,9\%, $18,4 \%, 17,9 \%, 11,47 \%$ e 9,7\%, do seu tempo para a documentação $11,15-17,20,21$.

Pode-se afirmar que existe preocupação dos enfermeiros com a documentação, entretanto, existe supervalorização do registro em livros secundários em detrimento dos registros no prontuário do cliente. Diversos estudos apontam que o enfermeiro tem utilizado o livro de ordens e ocorrências em substituição ao prontuário para o registro de intercorrências e avaliação do cliente ${ }^{22}$. Essa atitude caracteriza desobediência as legislações vigentes que determinam que o processo de cuidar deve ser registrado em prontuário ${ }^{23}$.

As atividades relacionadas ao domínio 7, Comunidade, não foram observadas durante o período de coleta de dados. Resultado semelhante foi encontrado em outro estudo que mostrou apenas uma intervenção relacionada a esse domínio ${ }^{15}$.

As atividades associadas são aquelas assumidas pela equipe de enfermagem, mas que poderiam ser realizadas por outras categorias profissionais. Essa categoria despendeu 6,2\% do tempo do enfermeiro. As atividades mais frequentes foram: fazer solicitações de suprimentos de rotina, sair da unidade para fazer encaminhamentos diversos, limpar e organizar armários e bancadas, receber e conferir materiais do estoque, conferência de prescrição médica, gerenciamento de leitos, organizar prontuários e fazer solicitações de consertos de manutenção, respectivamente.

Outros estudos mostram que o tempo dedicado às atividades associadas foram $12 \%, 10 \%, 7,7 \%, 7 \%, 6,5 \%, 5,3 \% \mathrm{e}$ 4,7\%, assemelhando-se ao estudo atual ${ }^{11,13,14-17,21}$

Na UTI investigada não existia enfermeiro para desempenhar as atividades de rotina relacionadas à organização e planejamento da assistência. A coordenadora do setor assumia a responsabilidade pela gestão de pessoas, de materiais e do ambiente. Assim, algumas atividades administrativas eram delegadas aos enfermeiros líderes, tais como solicitação de suprimento de rotina e conferência de materiais do estoque. 
Torna-se necessário repensar e redesenhar a delimitação das atividades e das atribuições a serem desempenhadas por enfermeiros gerentes, enfermeiros diaristas/rotinas, enfermeiros plantonistas/líderes e pela equipe técnica na UTI. A ausência de demarcação faz com que o enfermeiro assistencial assuma funções que são da competência dos enfermeiros gerentes. Embora, frequentemente, por necessidade do serviço, o enfermeiro seja obrigado a desenvolver atividades burocráticas no setor, isso não pode ser estipulado como atribuição habitual do enfermeiro líder. A realização destas atividades esporadicamente não descaracteriza o trabalho do enfermeiro na UTI, a problemática quase sempre está relacionada à frequência com que ele executa essas atividades, gerando sobrecarga do enfermeiro líder e distanciando-o de cuidados de forma direta aos clientes críticos.

A falta de profissionais administrativos na UTI apresenta-se como outro problema. O enfermeiro líder é responsável por atividades que não têm relação alguma com as suas atribuições legais e privativas, como por exemplo, organização de prontuários, gerenciamento de leitos e solicitações de consertos de manutenção.

As atividades pessoais corresponderam a $27,7 \%$ do tempo dos enfermeiros. As atividades predominantes foram descanso e socialização. Em estudos semelhantes o tempo dedicado as atividades pessoais foram $24 \%{ }^{16}, 18 \%{ }^{11}, 18 \%{ }^{13}$, $16,8 \%{ }^{15}, 16,4 \%{ }^{17}, 13 \%{ }^{21}, 7,21 \%^{20}$ e $3 \%{ }^{14}$, respectivamente.

Quanto à distribuição do tempo em cuidados diretos e indiretos, outros estudos desenvolvidos também mostraram que o enfermeiro despende maior tempo em atividades de cuidados indiretos. Estudos em Unidades de Saúde da Família verificaram que o tempo dedicado aos cuidados diretos e indiretos, pelos enfermeiros foram, respectivamente, $46 \%$ e $54 \%{ }^{14}$ e $52 \%$ e $48 \%{ }^{21}$. Em unidade médico-cirúrgica, verificou-se que $22 \%$ do tempo dos enfermeiros foram dedicados aos cuidados diretos e $50 \%$ aos cuidados indiretos. ${ }^{11} \mathrm{Em}$ unidade pediátrica e UTI neurológica o tempo dedicado pelos enfermeiros aos cuidados diretos e indiretos foram, respectivamente, $25,5 \%$ e $45,7 \%{ }^{16}$ e $20,5 \%$ e $55,4 \%{ }^{17}$

Em Unidade de emergência, a distribuição do tempo entre os cuidados prestados de forma direta e indireta foi proporcional, com $35 \%$ do tempo ${ }^{13}$. Estudo realizado em clínica médica, cirúrgica e UTI, verificou que os enfermeiros despenderam, respectivamente, 34,7\%, 35,7\% e 37,9\% de tempo em atividades de cuidados diretos e 43,8\%, 43,5\% e $40 \%$ em intervenções de cuidado indireto ${ }^{15}$.

Um estudo realizado em Portugal identificou que o enfermeiro despende o dobro do tempo nos cuidados indiretos, comparados aos cuidados diretos, resultado semelhante ao encontrado neste estudo ${ }^{24}$.

Estudo observacional conduzido em unidades de clínica médica, cirúrgica e especializada de um hospital de ensino mostrou que $58 \%$ das 90 horas observadas foram despendidas pelos enfermeiros executando atividades/intervenções de cuidado indireto ${ }^{25}$.

\section{Limitações do estudo}

A despeito da consistência do percurso metodológico, deve-se considerar como limitação da pesquisa, a ausência de um estudo piloto para testar o instrumento, a fim de restringir o número de intervenções segundo as características da população, tornar o instrumento mais objetivo e facilitar a sua aplicação.

\section{CONCLUSÃO}

O estudo permitiu a compreensão objetiva do gerenciamento do tempo dos enfermeiros na Unidade de Terapia Intensiva. Os resultados advindos da análise da distribuição do tempo dos enfermeiros em seu processo de trabalho apontam que $21,5 \%$ do tempo foram dedicados às intervenções de cuidados diretos, $44,7 \%$ aos cuidados indiretos, $6,1 \%$ às atividades associadas e $27,7 \%$ às atividades pessoais.

Analiticamente as intervenções/atividades mais frequentemente desenvolvidas pelos enfermeiros na UTI apontam para a utilização de maior parte de seu tempo em atividades não relacionadas ao cuidado direto ao paciente. Deve-se ressaltar as dificuldades vivenciadas pelos enfermeiros, tais como: número reduzido de profissionais para atender as demandas administrativas, excesso de atividades burocráticas e precariedade dos recursos materiais destinados à assistência.

Essas questões precisam ser discutidas, refletidas e pesquisadas não somente pelos enfermeiros, mas também pelos gestores das Instituições, pois esse cenário caótico requer a priorização de determinadas ações em detrimento de outras e gera desmotivação profissional. Nesse contexto, o enfermeiro enfrenta o desafio de gerenciar o seu tempo de forma que consiga atender a demanda de trabalho que lhe é imposta e manter a qualidade da assistência direta ao cliente.

Os achados podem ser utilizados pelos gestores para revisão e adequação do dimensionamento de profissionais na assistência direta e indireta e do processo de trabalho na UTI. Isso possibilitaria ao enfermeiro a oferta de uma assistência mais direta aos clientes criticamente enfermos. 
Acredita-se que esta investigação abre passagem para análises críticas e reflexivas, que versam sobre a distribuição do tempo dos enfermeiros em ambientes de alta complexidade. Por isso, a expectativa é que esses resultados auxiliem no avanço das discussões e em novas perspectivas analíticas neste campo do conhecimento.

\section{REFERÊNCIAS}

1. Hassard J. Tempo de trabalho: outra dimensão esquecida nas organizações. Traduzido por Rodrigues, Arakcy Martins. In: CHANLAT, Jean-François, coordenador. O indivíduo na organização: dimensões esquecidas. São Paulo: Atlas; 1992.

2. Mello MC, Fugulin FMT, Gaidzinski RR. Time in the health-related work process: a sociological approach. Acta paul. enferm. [Internet]. 2007 [cited 2020 Nov 16]; 20(1):87-90. DOI: https://doi.org/10.1590/S0103-21002007000100015

3. Rossi FR, S Maria Alice Dias da. Fundaments for managing process in care practices. Rev. esc. enferm. USP [Internet]. 2005 [cited 2021 Jan 15]; 39(4):460-8. DOI: https://doi.org/10.1590/S0080-62342005000400013

4. Marx K. O Capital. 14a ed. Rio de Janeiro: Bertrand; 1994.

5. Sanna MC. Work processes in Nursing. Rev. bras. enferm. [Internet]. 2007 [cited 2020 Nov 16]; 60(2):221-4. DOI: https://doi.org/10.1590/S0034-71672007000200018

6. Merhy EE, Onocko R. Agir em saúde: um desafio para o público. São Paulo: Hucitec; 1997.

7. Christovam BP, Porto IS, Oliveira DC. Nursing care management in hospital settings: the building of a construct. Rev. esc. enferm. USP [Internet]. 2012 [cited 2020 Nov 16]; 46(3):734-41. DOI: https://doi.org/10.1590/S0080-62342012000300028

8. Dochterman JMC, Bulechek GM. Classificações das Intervenções de enfermagem (NIC). 4aed. Porto Alegre: Artmed; 2008.

9. Silva SC, Padilha KG, Vattimo MFF. Enfermagem em Uti - Cuidando do Paciente Crítico. 2a ed. São Paulo: Manole, 2016.

10. Chaboyer W et al. A Comparison of Activities Undertaken by Enrolled and Registered Nurses on Medical Wards in Australia: An Observational Study. Int. J. Nurs. Stud. [Internet]. 2008 [cited 2020 Nov 16]; 45(9):1274-84. DOI: https://doi.org/10.1016/j.ijnurstu.2007.10.007

11. Bordin LC, Fugulin FMT. Nurses' time distribution: identification and analysis in a Medical-Surgical Unit. Rev. esc. enferm. USP [Internet]. 2009 [cited 2020 Nov 16]; 43(4):833-40. DOI: https://doi.org/10.1590/S0080-62342009000400014

12. Soares AVN, Gaidzinski RR, Cirico MOV. Nursing intervention identification in rooming-in. Rev. esc. enferm. USP [Internet]. 2010 [cited 2020 Dec 18]; 44(2):308-17. DOI: https://doi.org/10.1590/S0080-62342010000200010

13. Garcia EA, Fugulin FMT. Nurses' work time distribution at Emergency Service. Rev. esc. enferm. USP [Internet]. 2010 [cited 2020 Dec 18]; 44(4):1032-8. DOI: https://doi.org/10.1590/S0080-62342010000400025

14. Bonfim D, Gaidzinski RR, Santos FM, Gonçales CS, Fugulin FMT. The identification of nursing interventions in primary health care: a parameter for personnel staffing. Rev. esc. enferm. USP [Internet]. 2012 [cited 2020 Dec 18]; 46(6):1462-70. DOI: https://doi.org/10.1590/S0080-62342012000600025

15. Mello MC. Carga de trabalho de enfermagem: indicadores de tempo em unidades clínica, cirúrgica e terapia intensiva adulto [doctoral dissertation]. São Paulo: Universidade de São Paulo; 2011.

16. Andrade ACR. Distribuição do tempo de trabalho da equipe de enfermagem em unidade pediátrica [master's thesis]. São Paulo: Universidade de São Paulo; 2014.

17. Félix NN. Distribuição do tempo de trabalho da equipe de enfermagem em unidade de terapia intensiva neurológica [master's thesis]. São Paulo: Universidade de São Paulo; 2014.

18. Vasconcelos KO, Torres RSC, Silva SED, Baia RSM, Araujo JS, Cunha JO, et al. The importance of communication: family members of patients at an intensive care unit. Revista Conexao UEPG [Internet]. 2016 [cited 2021 Jan 10]; 12(2):196-207. DOI: https://doi.org/10.5212/Rev.Conexao.v.12.i2.0002

19. Carvalho V. Para uma Epistemologia da Enfermagem: Tópicos de Crítica e Contribuição. 1. ed. Rio de Janeiro: Corbã Gráfica; 2013.

20. Possari JF, Gaidzinski RR, Lima AFC, Fugulin FMT, Herdman TH. Use of the nursing intervention classification for identifying the workload of a nursing team in a surgical center. Rev. Latino-Am. Enfermagem [Internet]. 2016 [cited 2021 Jan 10]; 23(5):781-8. DOI: https://doi.org/10.1590/0104-1169.0419.2615

21. Bonfim D, Fugulin FMT, Laus AM, Peduzzi M, Gaidzinski RR. Time standards of nursing in Primary Health Care: an observational study. Rev. esc. enferm. USP [Internet]. 2016 [cited 2021 Mar 31]; 50(1):118-26. DOI: https://doi.org/10.1590/S0080623420160000100016

22. Pimpão FD, Filho WDL, Vaghetti HH, Lunardi VL. Nursing staff perception of nursing records: systematizing nursing care. Rev. enferm. UERJ [Internet]. 2010 [cited $2021 \mathrm{Apr} 02$ ]; 18(3):403-10. DOI: http://www.revenf.bvs.br/scielo.php?script=sci_arttext\&pid=S0104-35522010000300012\&lng=pt\&nrm=iso\&tlng=pt

23. CONSELHO FEDERAL DE ENFERMAGEM. Resolução no429, de 30 de Maio de 2012. Dispõe sobre o registro das ações profissionais no prontuário do paciente, e em outros documentos próprios da enfermagem, independente do meio de suporte - tradicional ou eletrônico. Diário Oficial da União, Brasília, DF, 8 Jun. 2012. Seção 1, p. 53.

24. Ribeiro OV, Vieira M, Cunha M, Dias A, Martins R. Time management in the planning of nursing cares. Revista Servir [Internet]. 2016 [cited 2021 Mar 20]; 59(4):7-11. DOI: https://repositorio.ipv.pt/handle/10400.19/4580

25. Campos MS, Oliveira BA, Perroca MG. Workload of nurses: observational study of indirect care activities/interventions. Revista Brasileira de Enfermagem [Internet]. 2018 [cited em 2021 Jun 16]; 71(2):297-305. DOI: https://doi.org/10.1590/0034-71672016-0561 